\title{
AVALIAÇÃO DA PRESENÇA DO POLIMORFISMO SstI DA APOLIPOPROTEÍNA C-III E SUAA ASSOCIAÇÃO COM OS NÍVEIS DE TRIGLICERÍDEOS EM UMA AMOSTRA DE DIABÉTICOS NO SUL DO BRASIL
}

\author{
EVALUATION OF THE PRESENCE OF SSTI POLYMORPHISM OF C-III APOLIPOPROTEIN AND ITS \\ ASSOCIATION WITH TRIGLYCERIDE LEVELS IN A DIABETIC SAMPLE FROM SOUTHERN BRAZIL
}

\author{
Bruna Cristina Jordona, Ivan Cunha Bustamante-Filho ${ }^{\mathrm{b}}$, Adriane Pozzobon ${ }^{\mathrm{c}}$ \\ abruna_jordon@hotmail.com, bivanbustamante@univates.br, cpozzobon@univates.br \\ Univates - Lajeado (RS), Brasil
}

Data de recebimento do artigo: 27/01/2015 Data de aceite do artigo: 29/05/2015

\section{RESUMO}

Introdução: $\mathrm{O}$ diabetes mellitus tipo 2 (DM2) é a doença metabólica mais comum. Objetivo: Pesquisar a presença do polimorfismo $S s t I$ da apolipoproteína C-III e verificar se existe associação com o perfil lipídico em uma amostra de diabéticos do Sul do Brasil. Materiais e Métodos: 65 pacientes com DM2 foram submetidos à avaliação antropométrica e análise do perfil lipídico e do polimorfismo SstI da APO C-III utilizando o método de reação em cadeia da polimerase seguida de digestão enzimática (PCR-RFLP). Foram classificados como alelo $\mathrm{G}$ os fragmentos que apresentaram tamanho de 269 e 159 pares de bases (pb) e como alelo C os fragmentos que apresentaram tamanho de $428 \mathrm{pb}$. Utilizou-se o teste t de Student para comparar as médias do perfil lipídico entre homens e mulheres, e a análise do polimorfismo foi feita por meio do teste qui-quadrado $\left(\chi^{2}\right)$ e do teste exato de Fischer. Resultados: A média de idade foi 64,67 $\pm 8,74$ anos e o IMC foi $31,09 \pm 4,86 \mathrm{~kg} / \mathrm{m}^{2} .55 \%$ dos indivíduos náo utilizaram medicaçáo antilipêmica. Os valores do colesterol total, triglicerídeos, HDL e LDL foram normais. Com relação ao polimorfismo, 80\% apresentou genótipo normal (CC), 3,1\% genótipo alterado (GG) e 16,9\% genótipo alterado (CG). Não foi observada associaçáo significativa entre o genótipo $\mathrm{CC}$ e os valores de triglicerídeos menores que $200 \mathrm{mg} / \mathrm{dL}$. Conclusáo: Na populaçáo avaliada a maioria dos indivíduos apresentou genótipo normal para o polimorfismo SstI sem haver associação com os níveis de triglicerídeos.

Palavras-chave: Apolipoproteína C; diabetes mellitus; triglicerídeos.

\section{ABSTRACT}

Introduction: Type 2 diabetes mellitus (DM2) is the most common metabolic disease. Objective: To investigate the presence of the $S s t I$ polymorphism of the C-III apolipoprotein and verify the association with the lipid profile in a diabetic sample from Southern Brazil. Materials and methods: Sixty-five patients with DM2 were subjected to anthropometric measurements, lipid profile and polymorphism analyses. The SstI polymorphism of APO C-III was made using the polymerase chain reaction method followed by enzymatic digestion (PCR-RFLP). Fragments that showed a size of 269 base pairs (bp) and $159 \mathrm{bp}$ were classified as $\mathrm{G}$ allele, and fragments that showed a size of $428 \mathrm{bp}$ were classified as $\mathrm{C}$ allele. We used the Student $t$ test to compare the means of the lipid profile between men and women while the polymorphism analysis was performed using the chi-square test $\left(\chi^{2}\right)$ and the Fisher exact test Results: The mean age was $64.67 \pm 8.74$ and BMI was $1.09 \pm 4.86 \mathrm{~kg} / \mathrm{m}^{2}$. Of the 65 individuals, $55 \%$ did not use antilipemic medication. The rate of total cholesterol, triglycerides, HDL and LDL levels were within normal limits. Regarding polymorphism, $80 \%$ of the subjects had normal genotype (CC), $3.1 \%$ changed genotype (GG), and $16.9 \%$ altered genotype (CG). There was no significant association between CC genotype and triglyceride levels below $200 \mathrm{mg} / \mathrm{dL}$. Conclusion: In the evaluated population, the majority of the individuals presented a normal genotype to the $S s t I$ polymorphism with no association with the triglycerides rate.

Keywords: Apolipoprotein C; diabetes mellitus; triglycerides 


\section{Introdução}

O diabetes mellitus (DM) é a doença metabólica mais comum, sendo usualmente classificado em: diabetes tipo 1 - DM1, no qual há deficiência completa de insulina, e diabetes tipo 2 - DM2, que se caracteriza por graus variados de diminuição de secreção e resistência ao hormônio pancreático insulina ${ }^{1}$. O DM acomete aproximadamente 250 milhóes de pessoas atualmente no mundo, sendo caracterizado por efeitos tardios e silenciosos que quando não são tratados interferem diretamente na qualidade de vida dos portadores ${ }^{2}$.

O DM está sendo englobado no grupo de grandes epidemias mundiais do século XXI; em 1995 já havia 135 milhóes de portadores, passando para 240 milhóes em 2005, e estima-se que irá atingir 366 milhōes em 2030, dos quais dois terços habitarão países em desenvolvimento ${ }^{3}$. O DM2 apresenta incidência elevada em indivíduos com mais de 40 anos de idade, associando-se à obesidade e à falta de atividade física. $\mathrm{Na}$ obesidade ocorre destruição das células beta do pâncreas, responsáveis pela produção da insulina, e devido à lipotoxicidade e ao desencadeamento de vias inflamatórias, favorece o desenvolvimento de DM2 $2^{4}$.

Um estudo realizado no Brasil no final da década de 1980 avaliou a população urbana de 30 a 69 anos e descobriu que a prevalência de DM era de 7,6\%, atingindo igualmente os dois sexos e aumentando com a idade e adiposidade corporal $^{5}$. As taxas mais elevadas foram vistas em cidades como Sáo Paulo (SP) e Porto Alegre (RS), evidenciando a influência da urbanização e da industrialização nessa patologia. Outro estudo relatou que a morbidade e a mortalidade causada pelo DM permanecem elevadas, mesmo existindo um controle dos fatores de risco convencionais, sugerindo que a fisiopatologia dessa doença envolve outros fatores de grande importância clínica, como a associação do DM com alterações no perfil lipídico ${ }^{6}$.

Dislipidemias são alteraçóes nas concentrações dos lipídeos no organismo, caracterizadas por distúrbios nos níveis de lipídios circulantes com ou sem repercussão sobre o território vascular associadas às manifestaçōes clínicas diversas e podendo ser influenciadas por distúrbios genéticos ou adquiridos ${ }^{7}$. Conforme a Organização Mundial de Saúde (OMS), a manutençáo dos níveis plasmáticos ideais de lipídeos constitui um dos maiores objetivos dentro do tratamento dos indivíduos diabéticos, justamente para evitar a progressão das complicações macrovasculares secundárias ao $\mathrm{DM}^{2}$.

Segundo Lima et al. ${ }^{8}$ as dislipidemias, hipertensão arterial sistêmica (HAS) e o DM são considerados importantes fatores no desenvolvimento da doença arterial coronariana (DAC), sendo a principal causa de morte de indivíduos adultos no mundo. Diversos estudos epidemiológicos têm demonstrado correlação positiva entre índices de glicemia elevados e altas concentraçóes plasmáticas de colesterol de lipoproteína de baixa densidade (LDL-C) e triglicerídeos e/ou baixas concentraçóes de colesterol de lipoproteína de alta densidade (HDL-C) e aumento de risco para DAC. O colesterol plasmático e os triglicerídeos são influenciados por vários fatores como: dieta, exercícios físicos, idade, sexo, raça e fatores genéticos.

As lipoproteínas de baixa densidade (LDLs) são consideradas as principais transportadoras plasmáticas de colesterol a todos os tecidos, sendo que sua absorção ocorre predominantemente no fígado (75\%), no tecido adiposo e nas glândulas adrenais ${ }^{10}$. A HDL possui inúmeras ações protetoras, como proteção antioxidante, mediação do fluxo de colesterol, inibição da expressão de moléculas de adesão celular, ativação de leucócitos, indução da produçáo de óxido nítrico $(\mathrm{NO})$, regulação da coagulação sanguínea e modulação da atividade plaquetária $^{11}$. Relatou-se em um estudo uma diminuição da concentração plasmática das HDLs e o aumento das LDLs com o desenvolvimento de DAC ${ }^{12}$.

As lipoproteínas são constituídas por um núcleo de lipídios neutros, não polares (ésteres de colesterol e triglicérides) e envolvidas por substâncias relativamente polares (fosfolipídios, colesterol livre e proteínas). Associado às lipoproteínas há o componente proteico denominado de apolipoproteínas (APOs), que são divididas em cinco grandes grupos e outros numerosos subgrupos: APO A (A-I, A-II, A-IV), APO B (B-100 e $\mathrm{B}-48)$ e APO C (C-I, C-II, C-III), que são as maiores constituintes do colesterol HDL, LDL e triglicerídeos, respectivamente ${ }^{7}$. Existem ainda a APO D e APO E, sendo que esses grupos diferem entre si em suas estruturas no comportamento físico-químico e em seus níveis plasmáticos ${ }^{10}$.

Em um estudo realizado por Lima et al. ${ }^{8}$, foi demonstrada associação positiva entre APO $\mathrm{B}$ e LDL e entre APO A-I e HDL, e ainda entre APO C e triglicerídeos. O gene APO C-III está localizado no cromossomo 11, sendo que vários polimorfismos foram identificados para esse gene. O polimorfismo mais estudado é o SstI, que ocorre devido à troca de uma citosina (C) por uma guanina $(\mathrm{G})$ na posição 3238 do nucleotídeo, na regiấo 3' não traduzida do gene. Essa substituição resulta na formação de dois alelos, $\mathrm{C} \mathrm{e} \mathrm{G}$, sendo o genótipo normal CC e o CG e GG alterados ${ }^{10}$. Fiegenbaum et al. demonstraram em um estudo que a presença desse sítio de restriçáo parece estar associada ao aumento nos níveis lipídicos em populaçóes de japoneses e indianos ${ }^{13}$.

Diante do exposto, o presente estudo teve como objetivo avaliar o perfil lipídico e verificar a prevalência do 
polimorfismo SstI da APO C-III em uma população de diabéticos no Sul do Brasil, bem como verificar se existe associação entre os níveis de triglicerídeos e o polimorfismo em questão.

\section{Metodologia}

O estudo proposto do tipo transversal avaliou 65 indivíduos com diabetes e hipertensão, usuários de Unidades Básicas de Saúde no interior do Rio Grande do Sul, cadastrados no programa SIS Hiperdia/MS da 16a Coordenadoria Regional de Saúde. Os critérios de exclusão foram: pacientes que apresentassem cardiopatias, hepatopatias, nefropatias, distúrbios de coagulação, doença infectocontagiosa conhecida, doença adrenal, anóes, deficientes mentais, mulheres grávidas e indivíduos com câncer. O estudo baseou-se nos princípios éticos contidos na Resolução 196/96 e na Declaração de Helsinki, tendo sido aprovado pelo Comitê de Ética em Pesquisa da Univates (Resolução CEP 017/11).

A avaliação antropométrica foi feita por meio do peso e da altura e cálculo de Índice de Massa Corporal (IMC), sendo que para os critérios de diagnóstico foram utilizados os parâmetros recomendados pela Organizaçáo mundial de saúde (OMS) ${ }^{14}$. Para as coletas, os participantes estavam em jejum de doze horas, sendo coletado $6 \mathrm{~mL}$ de sangue venoso. As amostras foram separadas ( $3 \mathrm{~mL}$ em cada) em tubos com anticoagulante EDTA para a realização da extração de DNA e tubo sem anticoagulante para determinação do Colesterol Total, HDL e triglicerídeos. O LDL foi determinado por meio da equação de Friedewald. Para a análise do perfil lipídico utilizou-se o método enzimático colorimétrico por meio dos kits comerciais da marca Bioclin $^{\oplus}$, e as leituras foram efetuadas em aparelho automatizado modelo BS120 da marca Mindray ${ }^{\circ}$. Antes das leituras serem realizadas foi utilizado um calibrador para testes analíticos em metodologias automatizadas e ainda controle patológico e normal para monitorar a exatidão e precisão dos resultados obtidos, sendo ambos os reagentes da marca Bioclin ${ }^{\curvearrowleft}$. Os valores de referência foram obtidos nas diretrizes brasileiras sobre dislipidemias demonstrando os seguintes valores: Colesterol total desejável de até $200 \mathrm{mg} / \mathrm{dL}$, limítrofe com valores de até $240 \mathrm{mg} / \mathrm{dL}$ e elevado com valores superiores a $240 \mathrm{mg} / \mathrm{dL}$; HDL desejável deve ser superior a $40 \mathrm{mg} /$ dL; LDL desejável de até $140 \mathrm{mg} / \mathrm{dL}$, limítrofe até 160 $\mathrm{mg} / \mathrm{dL}$ e elevado quando os valores estiverem superiores a $160 \mathrm{mg} / \mathrm{dL}$; e triglicerídeos desejável até $170 \mathrm{mg} / \mathrm{dL}$, limítrofe até $200 \mathrm{mg} / \mathrm{dL}$ e elevado quando estiver superior a $200 \mathrm{mg} / \mathrm{dL}^{15}$.

Para a detecção do polimorfismo Sst I do gene APO C-III foi realizada extração de DNA a partir de sangue periférico seguindo o protocolo de extração de DNA segundo Lahiri e Nurnberger ${ }^{16}$. A qualidade e a quantidade do DNA obtido foram avaliadas por meio da espectrofotometria nos comprimentos de onda 260 e 280 $\mathrm{nm}$. Para a avaliação da integridade do DNA foi feita a eletroforese em gel de agarose a $0,8 \%$. A eletroforese foi realizada em cuba horizontal utilizando tampão de corrida TBE (Tris-Ácido bórico-EDTA), agarose e brometo de etídio. Após a eletroforese as bandas de DNA foram visualizadas e capturadas pelo sistema de captura de imagem do equipamento Gel-Logic (Kodak $\left.{ }^{\odot}\right)$.

A identificação do polimorfismo SstI do gene APO C-III foi realizada pela reação em cadeia da polimerase (PCR), seguida de digestâo enzimática, utilizando os primers sense 5'GGT GAC CGA TGG CTT CAG TTC CCT GA 3' e antisense 5' CAG AAG GTG GAT AGA GCG CTG GCC $3^{\prime 10}$. As reações de PCR foram realizadas com um volume final de $50 \mu \mathrm{L}$, sendo utilizado PCR Buffer $(20 \mathrm{mM}$ de Tris HCL pH 8,4 e 50mM de KCL), 1,5 mM de $\mathrm{MgCl}_{2}, 1,25 \mathrm{U}$ de Taq DNA polimerase, $0,4 \mu \mathrm{M}$ de primers sense e antisense e $0,2 \mathrm{mM}$ de dNTP mix.

As amostras foram submetidas a um ciclo de 5 minutos a $95^{\circ}, 1$ minuto a $55^{\circ}$ e posteriormente a 35 ciclos de 1 minuto a $95^{\circ}, 40$ segundos a $58^{\circ}$ e 40 segundos a $72^{\circ}$, seguida de digestão enzimática com a enzima de restriçâo SstI por meio da técnica de RFLP (Polimorfismos de Fragmento de Restrição), na qual foi utilizado $10 \mathrm{uL}$ do produto de PCR, 5 unidades da enzima de restrição $S_{s t} I$ com incubação a $37^{\circ} \mathrm{C}$ overnight. Os fragmentos originados da digestão enzimática foram observados em gel de agarose a $2 \%$. Foram classificados como alelo $\mathrm{G}$ os fragmentos que apresentaram um tamanho de 269 pares de bases (pb) e $159 \mathrm{pb}$ e como alelo $\mathrm{C}$ os fragmentos que apresentaram um tamanho de $428 \mathrm{pb}^{10}$.

Os resultados das dosagens foram analisados estatisticamente, expressos como a média +/- desvio padrão da média (DP). Utilizou-se o teste $t$ de Student para comparaçáo das médias do perfil lipídico entre homens e mulheres. As diferenças entre as médias foram consideradas significativas quando os valores de $\mathrm{p}$ fossem $\leq 0,05$. A análise dos polimorfismos foi feita por meio do teste qui-quadrado $\left(\chi^{2}\right)$ para a frequência genotípica e o teste exato de Fischer foi utilizado para a frequência alélica. Utilizaram-se os softwares Biostat $5.0^{\circ}$ e Prism 5.0 grafic $\mathrm{pad}^{\circ}$. A presença do alelo $\mathrm{G}$ gera uma banda de $260 \mathrm{pb}$ e $159 \mathrm{pb}$, enquanto a presença do alelo $\mathrm{G}$ gera uma banda de $428 \mathrm{pb}$.

\section{Resultados}

Dos 65 indivíduos avaliados, $66,2 \%$ eram do sexo feminino e $33,8 \%$ do sexo masculino, sendo a média 
de idade de $64,67 \pm 8,74$ e o IMC de $31,09 \pm 4,86 \mathrm{~kg} / \mathrm{m}^{2}$, correspondendo à obesidade grau I. Não foram encontradas diferenças significativas no perfil lipídico entre os sexos (Tabela 1).

Tabela 1: Caracterização da amostra que avaliou a idade, IMC e perfil lipídico. Dados apresentados como média (x) e desvio padrão da média (DP).

\begin{tabular}{|c|c|c|c|}
\hline & \multirow{2}{*}{$\begin{array}{c}\text { Mulheres } \\
\mathrm{X} \pm \mathrm{DP} \\
\end{array}$} & \multicolumn{2}{|l|}{ Homens } \\
\hline & & $\mathrm{X} \pm \mathrm{DP}$ & $\mathbf{P}$ \\
\hline Idade & $64,90 \pm 8,64$ & $64,22 \pm 9,12$ & 0,7694 \\
\hline IMC & $31,78 \pm 5,26 \mathrm{~kg} / \mathrm{m}^{2}$ & $29,75 \pm 3,70 \mathrm{~kg} / \mathrm{m}^{2}$ & 0,1116 \\
\hline $\begin{array}{c}\text { Colesterol } \\
\text { total }\end{array}$ & $\begin{array}{c}169,11 \pm 37,18 \\
\mathrm{mg} / \mathrm{dL}\end{array}$ & $\begin{array}{c}179,40 \pm 38,28 \\
\mathrm{mg} / \mathrm{dL}\end{array}$ & 0,2997 \\
\hline Triglicerídeos & $\begin{array}{c}146,69 \pm 100,13 \\
\mathrm{mg} / \mathrm{dL}\end{array}$ & $\begin{array}{c}186,04 \pm 102,57 \\
\mathrm{mg} / \mathrm{dL}\end{array}$ & 0,3981 \\
\hline LDL & $95,32 \pm 35,72 \mathrm{mg} / \mathrm{dL}$ & $\begin{array}{c}102,08 \pm 31,22 \\
\mathrm{mg} / \mathrm{dL}\end{array}$ & 0,4724 \\
\hline HDL & $46,23 \pm 10,66 \mathrm{mg} / \mathrm{dL}$ & $\begin{array}{c}44,0 \pm 10,30 \mathrm{mg} / \\
\mathrm{dL}\end{array}$ & 0,4222 \\
\hline
\end{tabular}

IMC: índice de massa corporal; LDL: lipoproteína de baixa densidade; HDL: lipoproteína de alta densidade

Quanto ao uso de medicação, 3\% não faziam uso de nenhuma medicaçáo, $42 \%$ utilizavam medicamentos antilipêmicos (sinvastatina e estatina) e 55\% faziam uso de outros fármacos náo antilipêmicos. Comparando o grupo que tomava medicaçáo com o grupo sem medicação antilipêmica, não se observam diferenças estatísticas no perfil lipídico (Tabela 2).

Tabela 2: Perfil lipídico da população avaliada quanto ao uso de medicação antilipêmica. Dados apresentados como média (x) e desvio padrão da média (DP).

\begin{tabular}{cccc}
\hline & Com medicaçáo & \multicolumn{2}{c}{ Sem medicaçáo } \\
\hline & $\mathbf{X} \pm \mathrm{DP}$ & $\mathbf{X} \pm \mathrm{DP}$ & $\mathbf{P}$ \\
\hline Colesterol total & $\begin{array}{c}170,33 \pm 41,57 \\
\mathrm{mg} / \mathrm{dL}\end{array}$ & $\begin{array}{c}175,45 \pm 38,28 \\
\mathrm{mg} / \mathrm{dL}\end{array}$ & 0,60023 \\
& $172,14 \pm 184,39$ & $153,25 \pm 108,50$ & 0,6378 \\
Triglicerídeos & $\mathrm{mg} / \mathrm{dL}$ & $\mathrm{mg} / \mathrm{dL}$ & \\
& $85,02 \pm 43,45 \mathrm{mg} /$ & $99,30 \pm 36,27 \mathrm{mg} /$ & 0,1605 \\
LDL & $\mathrm{dL}$ & $\mathrm{dL}$ & \\
& $46,51 \pm 9,25 \mathrm{mg} /$ & $44,91 \pm 11,60 \mathrm{mg} /$ & 0,5575 \\
HDL & $\mathrm{dL}$ & $\mathrm{dL}$ & \\
\hline
\end{tabular}

LDL: lipoproteína de baixa densidade; HDL: lipoproteína de alta densidade.

Em relação ao polimorfismo, observou-se que $80 \%$ dos indivíduos apresentaram genótipo normal, ou seja, homozigoto (CC) e a proporção do genótipo alterado foi de $3,1 \%$ para o homozigoto (GG) e $16,9 \%$ de heterozigoto (CG). O número de alelos $\mathrm{C}$ foi 113 e o número de alelos $\mathrm{G}$ foi 17. A Figura 1 representa os genótipos analisados.

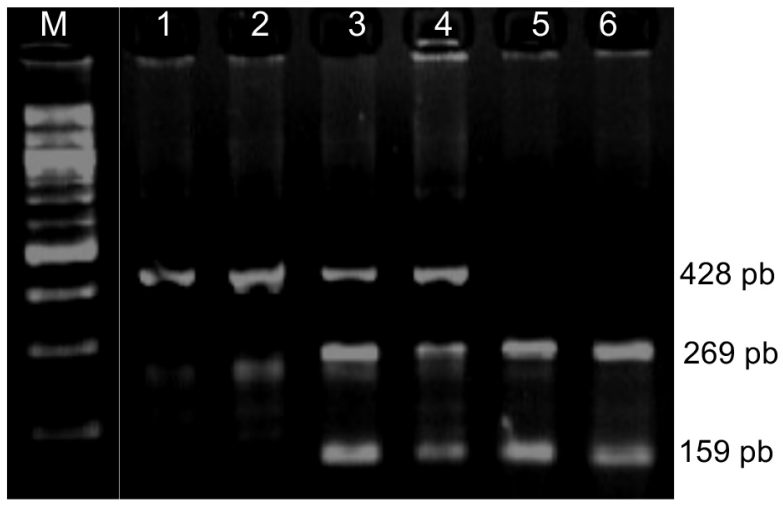

Figura 1: Gel de agarose $2 \%$ para a visualização dos genótipos. 1 e 2: fragmento de 428 pb correspondendo ao genótipo normal CC; 3 e 4: fragmentos de 428, 269 e 159 pb correspondendo ao genótipo GC; e 5 e 6: fragmentos de 269 e 159 pb correspondendo ao genótipo GG. $M=$ marcador de peso molecular $100 \mathrm{pb}$.

Para a análise do polimorfismo, a população em estudo foi subdividida em dois grupos, de acordo com os níveis de triglicerídeos. Para isso foram excluídos os indivíduos medicados. Associando o genótipo dos 38 indivíduos que não utilizaram medicação antilipêmica e os níveis de triglicerídeos, não se observou uma associação significativa entre o genótipo CC e os valores de triglicerídeos menores que $200 \mathrm{mg} / \mathrm{dL}\left(\chi^{2-} \mathrm{P}=0,2342\right)$ Em relação à frequência alélica também não houve diferença entre os valores (Teste exato de Fischer $=0,6538$ ). Os dados estão apresentados na Tabela 3.

Tabela 3: Frequência genotípica e fenotípica em relação aos triglicerídeos da população que não faz uso de fármacos antilipêmicos. Dados expressos em percentual.

\begin{tabular}{cccccc}
\hline \multicolumn{3}{c}{ Triglicerídeos na populaçáo que náo faz uso de } \\
antilipêmicos \\
\hline \multicolumn{3}{c}{ Frequência genotípica } & \multicolumn{3}{c}{ Frequência alélica } \\
\hline Grupo & $<\mathbf{2 0 0}$ & $>\mathbf{2 0 0}$ & & $<\mathbf{2 0 0}$ & $>\mathbf{2 0 0}$ \\
\hline CC & $65 \%$ & $13 \%$ & C & $73 \%$ & $13 \%$ \\
GG & $2,6 \%$ & $2,6 \%$ & G & $11,3 \%$ & $2,7 \%$ \\
CG & $16,8 \%$ & $0 \%$ & & & \\
\hline
\end{tabular}

\section{Discussão}

A hipertensão arterial sistêmica, as dislipidemias e o excesso de peso são importantes fatores de risco para o diabetes mellitus, pois anormalidades lipídicas em pacientes com DM são consequentes à resistência à insulina e caracterizadas por hipertrigliceridemia e níveis baixos de $\mathrm{HDL}^{17}$. 
No presente estudo observamos que a média dos valores do perfil lipídico da população em questão encontrava-se dentro dos valores normais, tanto no grupo que usou medicação quanto no grupo sem medicação. Em um estudo com uma populaçáo de diabéticos também não foi observado diferenças significativas entre os níveis plasmáticos de lipoproteínas de baixa densidade, colesterol total e triglicerídeos entre os sexos ${ }^{18}$. Além disso, todos os valores do perfil lipídico se encontraram dentro dos valores de referência. No entanto, o autor não menciona se a populaçáo estava ou não fazendo uso de medicação. Em contrapartida, em outro estudo foi possível verificar um aumento dos níveis de LDL e colesterol total em pacientes diabéticos ${ }^{8}$. Já os triglicerídeos e o HDL apresentaram valores normais; nesse caso, o uso de medicação antilipêmica também não foi citado no estudo. Outro estudo não encontrou diferença entre os sexos em relação à frequência de dislipidemias, porém foi observado que aproximadamente $70 \%$ dos pacientes de ambos os sexos possuíam pelo menos um tipo de alteração lipídica ${ }^{19}$.

Ressalta-se que a comparaçáo dos valores de prevalência de hiperlipidemia em diversos estudos é prejudicada em função das diferenças da população investigada e nos critérios utilizados para o diagnóstico das dislipidemias; além disso, observa-se na literatura grande variabilidade na ocorrência dessas alteraçôes no perfil lipídico ${ }^{20}$.

A apolipoproteína (APO) é o componente proteico das lipoproteínas, e as APOs participam, com os lipídios, fosfolipídios, colesterol, triglicerídeos e éster de colesterol na formação das classes de lipoproteínas. A APO C-III é componente da quilomicra, além de estar presente em pequena quantidade no HDL e LDL, sendo o fígado o principal local de síntese e, em menor grau, o intestino ${ }^{21}$. Lipoproteínas contendo APO C-III podem estar aumentadas no diabetes mellitus, obesidade visceral, resistência à insulina e dislipidemia ${ }^{22}$, sendo que as concentraçóes de APO C-III se correlacionam positivamente com os níveis de triglicerídeos em sujeitos normais e hipertrigliceridêmicos ${ }^{23}$. A deficiência do gene da APO C-III resulta em hipotrigliceridemia devido ao catabolismo das lipoproteínas, ricas em triglicérideos $^{24}$. Com relação ao diabetes, a APO C-III serve como um importante fator de risco, pois quando se encontra elevada afeta diretamente a funçáo e sobrevivência das células beta do pâncreas ${ }^{21,25}$.

Alelos variantes da região não codificante do gene da APO C-III (complexo AI-CIII-AIV) têm sido avaliados como marcadores genéticos de hipertrigliceridemia ${ }^{23}$. O SNP (polimorfismo de nucleotídeo único) C3238G, também chamado Sst I, tem sido associado com níveis plasmáticos aumentados de APO C-III e trigliceríde$\mathrm{os}^{26}$. No presente estudo, avaliou-se o polimorfismo SstI, sendo que a maioria da população analisada apresentou genótipo normal para esse polimorfismo, ou seja, $80 \%$ com o genótipo normal CC, 16\% com genótipo alterado CG e $4 \%$ com o genótipo alterado GG. Um estudo recente em uma população de pacientes com AIDS demonstrou que as frequências encontradas para o polimorfismo SstI da APO CIII foram de: 76,5\% (CC); $21,7 \%$ (CG), 1,8\% (GG) ${ }^{27}$.

As bases bioquímicas para a associação entre o alelo $\mathrm{G}$ e a hipertrigliceridemia ainda não estão bem esclarecidas. Um estudo demonstrou associação entre níveis de APO C-III e o alelo G em uma população japonesa. Além disso, nesse mesmo estudo encontrou-se uma alta prevalência do alelo $G$, sendo muito mais frequente do que quando comparado com a populaçáo caucasiana ${ }^{10}$. Também não foi encontrada diferença nas frequências genotípicas do gene da APO C-III entre os grupos de indivíduos com doença arterial coronariana prematura em um estudo de caso e controle, e náo houve associação desses grupos entre os genótipos e os níveis de triglicerídeos ${ }^{29}$. Existem poucos estudos na literatura que relacionam esse polimorfismo com nos níveis de triglicerídeos, e os poucos estudos existentes apresentam dados divergentes conforme as populaçóes analisadas; portanto, o polimorfismo $S s t I$ da APO C-III pode estar relacionado com os níveis de triglicerídeos em determinadas populaçóes.

\section{Conclusão}

$\mathrm{Na}$ amostra avaliada, a maioria dos indivíduos apresentou genótipo normal para o polimorfismo SstI sem haver diferenças no perfil lipídico, assim como não houve associação entre os níveis de triglicerídeos e o polimorfismo SstI. Estudos prospectivos de longo prazo com um número maior de indivíduos diabéticos podem contribuir para melhor avaliação dos mecanismos genéticos envolvidos com o diabetes mellitus e as dislipidemias.

\section{Agradecimentos}

Aos Laboratórios de Biologia Molecular e Bioquímica da Univates.

\section{Referências}

1. Gross JL, Silveiro SP, Camargo JL, Reicheltz AJ, Azevedo MJ. Diabetes melito: diagnóstico, classificação e avaliação do controle glicêmico. Arq Bras Endocrinol Metab. 2002;46(1):16-26. 
2. World Health Organization. Diabetes mellitus fact sheets. 2002 [acesso em 2011 Set 5]. Disponível em: http://www. who.int/ inf-fs/en/fact138.html

3. Wild S, Roglic G, Green A, Sicree R, King H. Global prevalence of diabetes: estimates for the year 2000 and projections for 2030. Diabtes Care. 2004;27(5):1047-53.

4. Wellen KE, Hotamisligil GS. Inflammation, stress and diabetes. J Clin Invest. 2005;115:1111-9.

5. Malerbi DA, Franco LJ. Multicenter study of the prevalence of diabetes mellitus and impaired glucose tolerance in the urban Brazilian population aged 30-69yr. The Brazilian cooperative group on the study of diabetes prevalence. Diabetes care. 1992;15(11):1509-16.

6. Yamada T, Sato A, Nishimori T, Mitsuhashi T, Terao A, Sagai $\mathrm{H}$, et al. Importance of hypercoagulability over hyperglycemia for vascular complication in type 2 diabetes. Diabetes Res Clin Prac. 2000;49(1):23-31.

7. Mcqueen MJ, Hawken S, Wang X, Ounpuu S, Sniderman A, Probstfield J, et al. Lipids, lipoproteins and apolipoproteins as risk markers of myocardial infarction in 52 countries. Lancet. 2008;372(9634):224-33.

8. Lima LM, Carvalho MDG, Soares AL, Lasmar MC, Novelli BA, Sousa MO. Correlação entre os níveis plasmáticos de apolipoproteínas A-I e B e o perfil lipídico em indivíduos com e sem diabetes mellitus tipo 2 e hipertensão arterial. J Bras Patol Med Lab. 2005;41(6):411-7.

9. Motta VT. Bioquímica clínica para o laboratório: princípios e interpretaçóes. São Paulo: Missau; 2003.

10. Parzianello L, Oliveira G, Coelho JC. Apolipoprotein CIII polymorphism and triglyceride levels of a Japanese population living in the southern Brazil. Braz J Med Biol Res. 2008;41(6):462-7.

11. Lima E, Couto D. Estrutura, metabolismo e funções fisiológicas da lipoprotepina de alta densidade. J Bras Patol Med Lab. 2006;42(3):169-178.

12. Young CE, Karas RH, Kuvin JT. High-density lipoprotein cholesterol and coronary heart disease. Cardiol Rev. 2004;12(2):107-19.

13. Fiegenbaum M, Andrade FM de, Hutz MH. Association between plasma lipid parameters and APOC3 genotypes in Brazilian subjects: effect of gender, smoking and APOE genotypes. Clin Chim Acta. 2007;380(1-2):175-81.

14. World Health Organization. Obesity Preventing and managing the Global Epidemic. Report of a WHO Consultation on Obesity. Geneva: WHO; 1998.

15. BRASIL. IV Diretrizes brasileiras sobre dislipidemias e diretriz de prevenção da aterosclerose do departamento de aterosclerose da sociedade brasileira de cardiologia. Arquivo brasileiro de cardiologia. São Paulo; 2007.
16. Lahiri DK, Nurnberger JIJR. A rapid non-enzymatic method for the preparation of HMW DNA from blood for RFLP studies. Nucleic Acids Research. 1991;19(19):5444.

17. Souza LJ, Chalita FEB, Reis AFF, Teixeira CL, Gicovate Neto C, Bastos DA, et al. Prevalência de diabetes mellitus e fatores de risco em Campos dos Goytacazes, RJ. Arq Bras Endocrinol Metab. 2003;47(1):69-74.

18. Mota APL, Carvalho MdasG, Lima LM, Santos MERdeC, Sousa MdeO. Lipoproteína (a) em pacientes portadores de doença arterial obstrutiva periférica e/ou diabetes mellitus tipo 2. J Bras Patol Med Lab. 2008;44(2):89-95.

19. Vasques ACJ, Pereira PF, Gomide RM, Batista MCR, Campos MTFS, Sant'Ana LFR, et al. Influência do excesso de peso corporal e da adiposidade central na glicemia e no perfil lipídico de pacientes portadores de diabetes mellitus tipo 2. Arq Bras Endocrinol Metab. 2007;51(9).

20. Bevilacqua MR, Gimeno SGA, Matsumura LK, Ferreira SRG. Hiperlipidemias e fatores dietéticos: estudo transversal entre nipo-brasileiros. Arq Bras Endocrinol Metab. 2007;51(4).

21. Novak EM, Bydlowski PS. Biologia molecular nas dislipidemias. Arq Bras Cardiol. 1996;67(6).

22. Haynes W. Triglyceride-rich lipoproteins and vascular function. Arteriosc Thromb Vasc Biol. 2003;23(2):153-5.

23. Batal R, Tremblay M, Barrett PH, Jacques H, Fredenrich A, Mamer O, et al. Plasma kinetics of apoC-III and apoE in normolipidemic and hypertriglyceridemic subjects. J Lipid Res. 2000;41:706-18.

24. Maeda N, Li H, Lee D, Oliver P, Quarfordt SH, Osada J. Targeted disruption of the apolipoprotein C-III gene in mice results in hypotriglyceridemia and protection from postprandial hypertriglyceridemia. J Biol Chem. 1994;269:23610-6.

25. Holmberger R, Refai E, Höög A, Crooked RM, Grahamd $\mathrm{M}$, Olivecronae $\mathrm{G}$, et al. Lowering apolipoprotein CIII delays onset of type 1 diabetes. Proc Natl Acad Sci USA. 2011;108(26):10685-9.

26. Shoulders CC, Grantham TT, North JD. Hypertriglyceridemia and the apolipoprotein CIII gene locus: lack of association with the variant insulin response element in Italian school children. Hum Genet. 1996;98:557-66.

27. Pezzi N, Bazzo KO, Matte MCC, Fiengenbaum M. Influência de polimorfismos nos genes APO CIII e CYP2B6 no perfil lipídico de pacientes HIV positivos sob terapia antirretroviral. Arq Bras Cardiol. 2011;97(2):279-85.

28. Izar MC, Helfenstein FA, Ihara SSM. Fatores de risco, marcadores bioquímicos e polimorfismos genéticos na doença arterial coronariana prematura. Arq Bras Cardiol. 2003;80(4):379-87.

\section{Como citar este artigo:}

Jordon BC, Bustamante Filho IC, Pozzobon A. Avaliação da presença do polimorfismo da apolipoproteína c-III e sua associação com os níveis de triglicerídeos em uma amostra de diabéticos no Sul do Brasil. Rev. Aten. Saúde. 2015;13(46):72-7 